\title{
Malayan Pit Viper Envenomation and Treatment in Thailand
}

\author{
Thunyaporn Tangtrongchitr, \\ Suthimon Thumtecho (D) ${ }^{1,3}$ \\ Jiratchaya Janprasert ${ }^{4}$ \\ Kitisak Sanprasert ${ }^{2}$ \\ Achara Tongpoo' \\ Yuvadee Tanpudsa' \\ Satariya Trakulsrichai $\mathbb{D D}^{1,5}$ \\ Winai Wananukul ${ }^{1,6}$ \\ Sahaphume Srisuma $\mathbb{D}^{1,6}$
}

'Ramathibodi Poison Center, Faculty of Medicine Ramathibodi Hospital, Mahidol University, Bangkok, Thailand;

${ }^{2}$ Department of Trauma and Emergency Medicine, Phramongkutklao Hospital,

Bangkok, Thailand; ${ }^{3}$ Division of

Toxicology, Department of Medicine,

Chulalongkorn University and King

Chulalongkorn Memorial Hospital, the

Thai Red Cross Society, Bangkok,

Thailand; ${ }^{4}$ Faculty of Medicine

Ramathibodi Hospital, Mahidol

University, Bangkok, Thailand;

${ }^{5}$ Department of Emergency Medicine,

Faculty of Medicine Ramathibodi

Hospital, Mahidol University, Bangkok,

Thailand; ${ }^{6}$ Division of Clinical

Pharmacology and Toxicology,

Department of Medicine, Faculty of

Medicine Ramathibodi Hospital, Mahidol

University, Bangkok, Thailand
Correspondence: Sahaphume Srisuma Tel +66 2201 1682

Email boat_ra_ac@hotmail.com
Introduction: The Malayan pit viper (MPV; Calloselasma rhodostoma) is a hematotoxic snake found in all regions of Thailand and many countries in Southeast Asia. Treatment of MPV envenomation varies among facilities due to their capabilities.

Materials and Methods: This study was a retrospective review of patients with MPV envenomation who were reported to the Ramathibodi Poison Center from 1 July 2016 to 30 June 2018.

Results: Of the 167 patients (median age, 40.5 years; range, $1.3-87.0$ years) bitten by an MPV, the most common bite site was the foot (29.3\%). Most patients reached the hospital within 1 hour of being bitten. One-hundred fifty-six patients $(93.4 \%)$ had local effects from envenomation; 17 patients $(10.2 \%)$ had severe local complications including necrotizing fasciitis (3.0\%) and compartment syndrome (7.2\%). Systemic effects such as hemorrhage and abnormal hemostasis occurred in 147 patients $(88.0 \%)$. Additional effects included abnormal venous clotting time in 123 patients $(73.7 \%)$, unclotted 20 -minute whole blood clotting time in 57 patients $(34.1 \%)$, low platelet counts $(<50,000 / \mu \mathrm{L})$ in 29 patients $(17.4 \%)$, prolonged international normalized ratio $(>1.2)$ in 51 patients $(30.5 \%)$, and systemic bleeding in 14 patients $(8.4 \%)$. The median onset of bleeding disorder was 6 hours. Monitoring for 24, 48, and 49 hours after bite enabled detection of systemic effects in $94.2 \%, 99.3 \%$, and $100.0 \%$, respectively. Three hundred fifteen courses of antivenin were administered to 144 patients $(86.2 \%)$. All the patients who received antivenin recovered from bleeding disorder. Only $7.0 \%$ of antivenin doses were administered without Thai Red Cross indications. Allergic reactions from antivenin occurred in $34.7 \%$ of the 144 patients. One hundred thirty patients $(77.8 \%)$ received antibiotics, and 32 patients (19.2\%) required surgical management, including debridement and fasciotomy.

Conclusion: MPV envenomation results in local and systemic effects. Most systemic effects were abnormal clotting test results. Most patients reported onset of bleeding disorder within 48 hours.

Keywords: Malayan pit viper, snake venom, hematotoxin, antivenin, onset of abnormality

\section{Introduction}

Snakebite is a public health problem worldwide, which is more common in developing countries. Thailand has an abundance of venomous snakes, including both neurotoxic and hematotoxic snakes. Malayan pit vipers (MPVs; Calloselasma rhodostoma) are common hematotoxic snakes found in Thailand; ${ }^{1}$ these snakes are a major cause of snake envenomation in Southeast Asia, and chronic disability resulting from local necrosis can be considerable. To the best of our knowledge, there have been few studies of MPV envenomation in Southeast Asia. ${ }^{2-4}$ 
MPV envenomation can cause serious disability and death. $^{3,5}$ Clinical manifestations of MPV bite include local effects, systemic effects, or both local and system effects. Local effects are characterized by swelling, discoloration, blistering, or necrosis. Systemic toxicity comprises bleeding disorder, which can range from abnormal clotting tests to hemorrhagic syndrome. ${ }^{6}$ The main mechanism underlying the hematologic effects of MPV envenomation is consumptive coagulopathy by thrombinlike enzymes. ${ }^{7}$ Additional biological constituents of MPV venom include hemorrhagin, platelet aggregation inducer, disintegrin, antiplatelet protease, $\alpha$-fibrinogenase, L-amino acid oxidase, etc. ${ }^{6,8}$ Species-specific antivenin reverses systemic effects by neutralization of circulating venom. Queen Saovabha Memorial Institute, Thai Red Cross Society produces MPV antivenin and lists the indications for its use. In Thailand, there are varied approaches to management and monitoring of MPV-bitten patients, because of limited facility capabilities (eg, laboratory tests and antivenin).

Indications for administration of antivenin, as recommended by Queen Saovabha Memorial Institute, are as follows: (1) venous clotting time (VCT) $>20$ minutes; (2) unclotted 20-minute whole blood clotting time; (3) platelet count $<50,000 / \mu \mathrm{L}$; (4) international normalized ratio (INR) $>1.2$; (5) systemic bleeding; (6) compartment syndrome requiring fasciotomy. ${ }^{9}$ The recommended dose is 3-5 vials per dose; the duration of infusion is 30-60 minutes.

The previous studies of MPV bites in Thailand did not report the onset of systemic toxicity or the results of follow-up clotting tests and did not include all regions of the country. ${ }^{3,5,10}$ Therefore, the primary objective of this study was to characterize MPV envenomation in Thailand included demographic data, clinical effects, treatments, complications, and medical outcome. Secondary objective was to demonstrate earliest and latest onset of hematotoxicity.

\section{Materials and Methods}

\section{Patients, MPV Bite Confirmation, and Data Collection}

This was a retrospective descriptive study of MPV-bitten patients reported to the Ramathibodi Poison Center (RPC) from 1 July 2016 to 30 June 2018. The RPC is the first poison control center in Thailand, which has served the entire Thai population of all regions for more than 24 years. More than 20,000 consultation calls are received each year. RPC supports medical information, and also gives suggestions on diagnosis and management. Follow-up calls to hospitals where the patients are undergoing treatment are made to provide ongoing recommendations and determine medical outcomes until affected patients are discharged from the hospital. The decisions on patient management are finalized by the primary team who took care the patients. When MPV bitten patients had indication for antivenin, RPC primarily suggests using monovalent MPV antivenin. Regarding the numbers and types of antivenins stocked in the hospital, sometimes the given antivenin may be polyvalent hematotoxin snake antivenin. All poisoning data are recorded in the RPC database. Ethical approval for the study was obtained from the Institutional Ethics Committee Board of Ramathibodi Hospital Faculty of Medicine, Mahidol University. The Ethics Approval Reference Number is MURA 2018/924. As a retrospective study of deidentified data from existing database, the requirement of patient's informed consent document was waived by the Institutional Ethics Committee Board. Institutional consent and approval were obtained from Faculty of Medicine Ramathibodi Hospital, Mahidol University, prior to study commencement.

Diagnoses of MPV bites were confirmed by one of the following methods: (1) the biting snake was caught and brought to the hospital; (2) the patient was able to recognize the type of snake; (3) the snakebite was unidentified but resulted in hematologic symptoms that responded to MPV antivenin. If the primary care teams acquired photos of the snake, those photos were electronically sent to the RPC for snake identification.

The following data were reviewed in this study: demographic data (age, sex, region, time of bite, bite sites, time to hospital, underlying disease, and current medications), clinical effects, complications, treatment (antivenin and adverse reaction, surgical management, and antibiotics), and recovery time.

\section{Definitions}

Dry bites were defined as venomous snakebites that did not result in any symptoms. ${ }^{7}$

Patients were diagnosed with anaphylaxis reaction when they developed two or more of the following types of symptoms: skin-mucosal, respiratory, cardiovascular, and gastrointestinal. Patients with allergic reaction were 
divided into groups according to onset following exposure to snake venom or to antivenin.

The degree of swelling was categorized into seven grades: 1, local swelling; 2, swelling in one joint; 3, swelling in $>1$ joint; 4 , swelling in two joints; 5 , swelling in $>2$ joints; 6, swelling in three joints; and 7, swelling in $>3$ joints. $^{11}$

Onset of systemic effects refers to bleeding or abnormal laboratory results that occurred after initial evaluation and initial normal clotting test results. Cumulative incidences of systemic toxicities were evaluated at each time point. If the patients first presented at the hospital later than 24 hours after bitten, they were excluded from cumulative incidence analysis.

Appropriate use of antivenin was regarded as administration with the correct indication, dose, and infusion duration, based on Queen Saovabha Memorial Institute recommendations.

Anaphylaxis or early anaphylactic reactions from venom and antivenin were referenced from the National Institute of Allergy and Infectious Disease and the Food Allergy and Anaphylaxis Network. ${ }^{12}$

Recurrence of systemic toxicity was defined as the presence of bleeding disorders that met the indication for antivenin, after initial recovery.

Full recovery was defined as the absence of bleeding and the presence of normal clotting test results at the time of discharge.

\section{Statistical Analysis}

All statistical analyses were performed with Microsoft Excel for Mac, version 16.16.17. Data were analyzed and presented as descriptive statistics with median, interquartile range $(\mathrm{IQR})$, and range ( $\min -\max )$.

\section{Results}

\section{Patient Characteristics}

During the study period, 167 MPV-bitten patients were reported to the RPC; 105 (62.9\%) were male patients. Median patient age was 40.5 years (IQR, 28.5-56; range, 1.3-87) (Table 1). Seventeen patients (10.2\%) were children aged $<15$ years. MPV bites occurred in all regions of Thailand. Most cases were reported from the southern region (Figure 1A) and were bitten during the rainy season (Figure 1B). Patients were most commonly bitten at 8 p.m. (Figure 1C). The median time from the bite to arrival at
Table I Characteristics of Patients with Malayan Pit Viper Envenomation Reported to Ramathibodi Poison Center

\begin{tabular}{|l|c|}
\hline Characteristic & n (\% of I67 Patients) \\
\hline Age [years, median (IQR; & $40.5(28.5-56 ;$ I.3-87) \\
range)] & \\
\hline Gender & $105(62.9)$ \\
Male & $62(37.1)$ \\
Female & \\
\hline Bitten area & $38(22.8)$ \\
Finger & $18(10.8)$ \\
Toe & $14(8.4)$ \\
Hand & $49(29.3)$ \\
Foot & $1(0.6)$ \\
Wrist & $26(15.6)$ \\
Ankle & $2(1.2)$ \\
Arm & $11(6.6)$ \\
Leg & I (0.6) \\
Shoulder & $1(0.6)$ \\
Buttock & I (0.6) \\
Multiple site & \\
\hline Diagnosis of MPV bite & $96(57.5)$ \\
Brought snake to hospital & $56(33.5)$ \\
Able to recognized the type of & \\
snake & $15(9.0)$ \\
Unknown snakebites which & \\
response to MPV antivenin & \\
\hline
\end{tabular}

Abbreviations: MPV, Malayan pit viper; IQR, interquartile range.

a health-care facility was 1 hour (IQR, 30 minutes to 2 hours; range, 5 minutes to 7 days).

Ninety-six patients $(57.5 \%)$ brought MPVs or MPV carcasses to the hospital. Fifty-six patients (33.5\%) recognized MPVs at the time of the bit. Fifteen patients $(9.0 \%)$ experienced unknown snakebites, which resulted in bleeding disorders that were alleviated by MPV antivenin (Table 1).

\section{Clinical Effects}

The most common area bitten was the foot (49 patients, 29.3\%) (Table 1). Fang marks were identified in 107 patients (64.1\%). One-hundred fifty-six patients $(93.4 \%)$ experienced local effects. Fifteen patients $(9.0 \%)$ experienced local effects without any systemic effects (Table 2). Grade 1 swelling was the most common severity of local swelling (33.5\%) (Figure 2). Local pain was reported in 79 patients $(47.3 \%)$. Local complications included compartment syndrome (12 patients, 7.2\%) and necrotizing fasciitis (five patients, $3.0 \%$ ). However, no wound or blood cultures were reported. 
A

Cases (n)

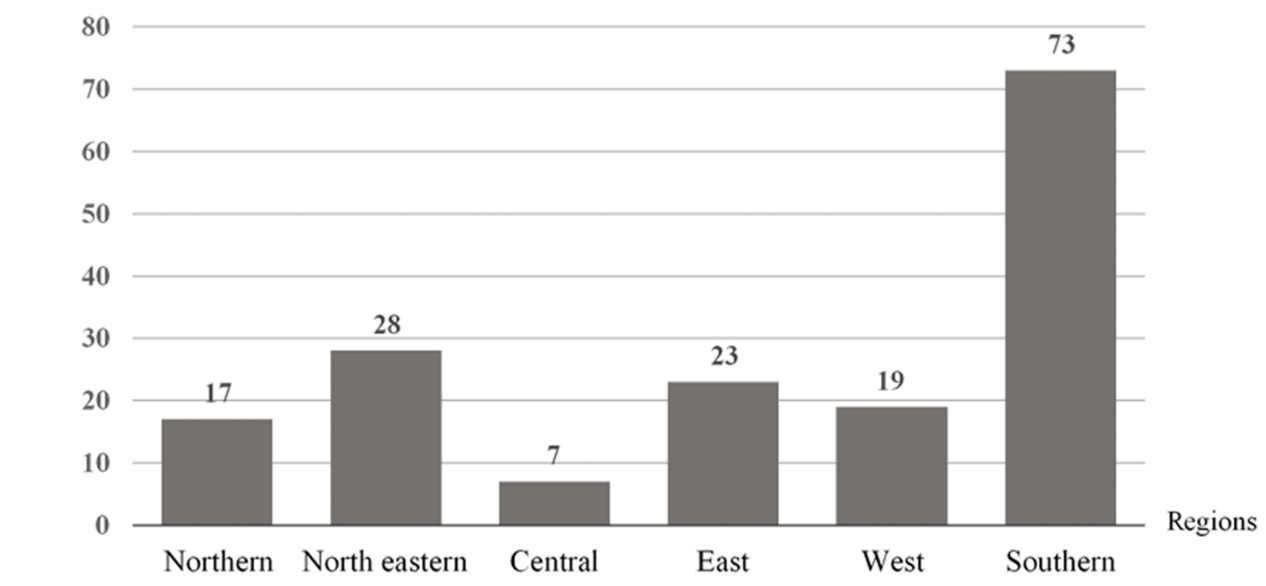

B
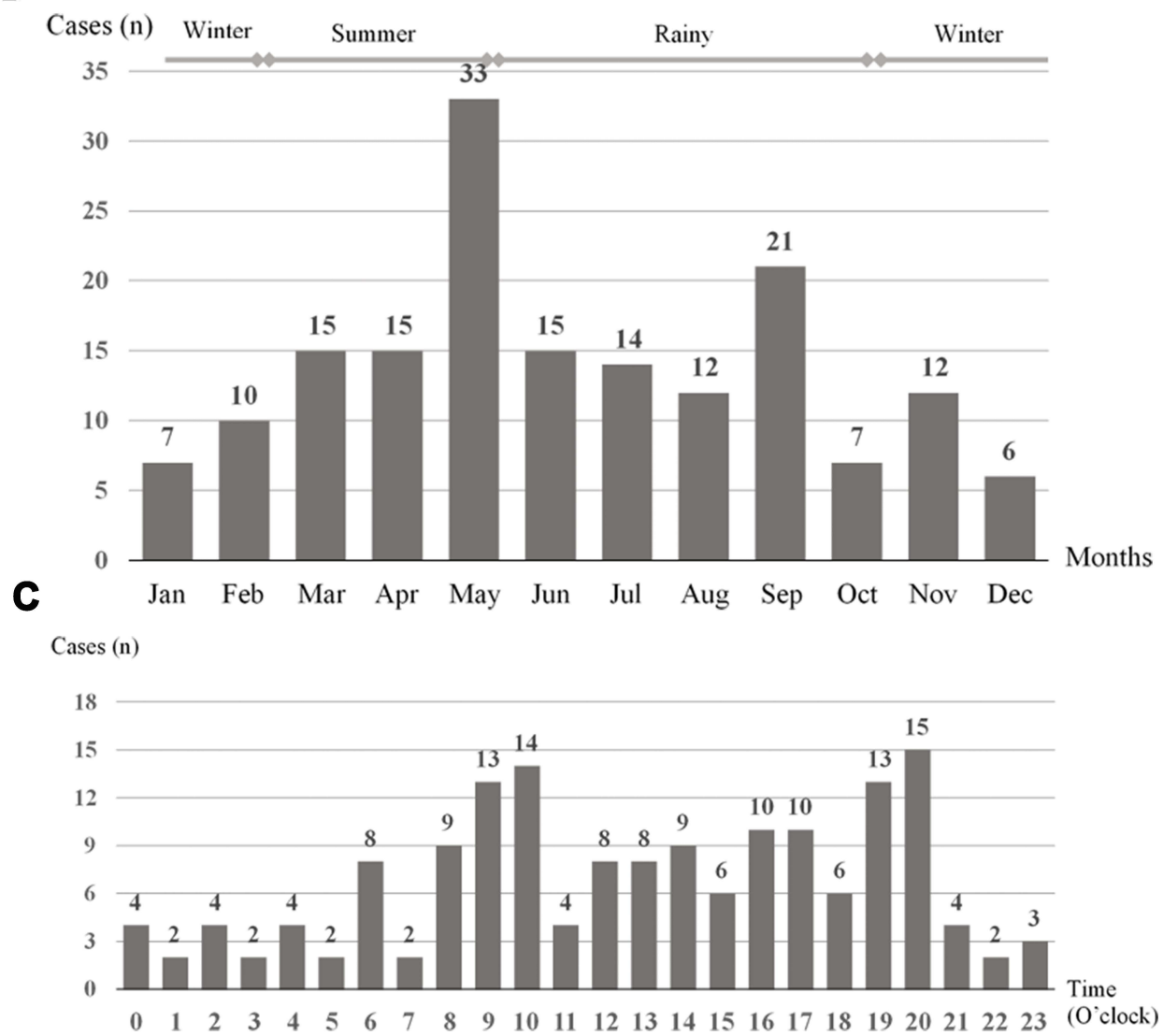

Figure I Patients with Malayan pit viper bite reported to Ramathibodi Poison Center during July 2016 to June 2018. (A) Malayan pit viper bites by region of Thailand. (B) Malayan pit viper bites by month and season. (C) Malayan pit viper bites by time of day (O'clock).

Systemic effects occurred in 147 patients (88.0\%). Six patients experienced systemic effects without local effects. Five patients exhibited dry bites. VCT was the most common test performed (Table 3). Systemic bleeding occurred in 14 patients $(8.4 \%)$. Types of bleeding included gingival (11 patients), vaginal (two patients), and hematemesis (four patients).

The median durations until onset of each abnormal test result and systemic bleeding are shown in Table 4. The median onset of bleeding disorder was 6 hours (IQR, 1.5- 
Table 2 Clinical Manifestations of Patients with Malayan Pit Viper Envenomation Reported to Ramathibodi Poison Center

\begin{tabular}{|l|c|}
\hline Clinical Manifestations & n (\% of I67 Patients) \\
\hline Local effect & $156(93.4)$ \\
Swelling & $149(89.2)$ \\
Local pain & $79(47.3)$ \\
Bleeding per wound & $33(19.8)$ \\
Ecchymosis & $32(19.2)$ \\
Bleb & $38(22.8)$ \\
Necrosis & $22(13.2)$ \\
Hematoma & $7(4.2)$ \\
Necrotizing fasciitis & $5(3.0)$ \\
Compartment syndrome & $12(7.2)$ \\
\hline Systemic effect & $147(88.0)$ \\
Prolonged VCT & $123(73.7)$ \\
Unclotted 20WBCT & $57(34.1)$ \\
Platelet <50,000/mcL & $29(17.4)$ \\
INR >I.2 & $5 I(30.5)$ \\
Systemic bleeding & $14(8.4)$ \\
\hline
\end{tabular}

Abbreviations: MPV, Malayan pit viper; VCT, venous clotting time; $20 \mathrm{WBCT}, 20-$ minute whole blood clotting test; INR, international normalized ratio.

Table 3 Number of Patients with Laboratory Tests and Patients with Abnormal Results $(n=167)$

\begin{tabular}{|l|c|c|}
\hline $\begin{array}{l}\text { Clotting } \\
\text { Test }\end{array}$ & $\begin{array}{c}\text { No. Cases with } \\
\text { Laboratory } \\
\text { Test }\end{array}$ & $\begin{array}{c}\text { No. Cases with Abnormal } \\
\text { Result (\% of Cases with Lab } \\
\text { Test) }\end{array}$ \\
\hline VCT & 152 & $123(80.9)$ \\
20WBCT & 99 & $57(57.6)$ \\
Platelet & 162 & $29(17.9)$ \\
INR & 116 & $51(44.0)$ \\
\hline
\end{tabular}

Abbreviations: VCT, venous clotting time; $20 \mathrm{WBCT}, 20$-minute whole blood clotting test; INR, international normalized ratio.

Table 4 Onset of Systemic Toxicity in Patients with Malayan Pit Viper Envenomation Reported to Ramathibodi Poison Center

\begin{tabular}{|l|c|}
\hline Systemic Effects & $\begin{array}{c}\text { Median Onset of } \\
\text { Abnormality (IQR; Range) }\end{array}$ \\
\hline Prolonged VCT & $6.5 \mathrm{~h}(2.75-13 \mathrm{~h} ; 30 \mathrm{~min}-49 \mathrm{~h})$ \\
Unclotted 20WBCT & $13 \mathrm{~h}(7-13 \mathrm{~h} ; 10 \mathrm{~min}-66.5 \mathrm{~h})$ \\
Platelet < 50,000 mcL & $3 \mathrm{~h}(\mathrm{I}-9.5 \mathrm{~h} ; 30 \mathrm{~min}-18 \mathrm{~h})$ \\
INR > I.2 & $9 \mathrm{~h}(5-18.5 \mathrm{~h} ; 30 \mathrm{~min}-96 \mathrm{~h})$ \\
Systemic bleeding & $5.5 \mathrm{~h}(4.1-22.4 \mathrm{~h} ; 15 \mathrm{~min}-59 \mathrm{~h})$ \\
Overall onset of systemic effects & $6 \mathrm{~h}(1.5-12 \mathrm{~h} ; 10 \mathrm{~min}-96 \mathrm{~h})$ \\
\hline
\end{tabular}

Abbreviations: VCT, venous clotting time; $20 \mathrm{WBCT}, 20$ minutes whole blood clotting test; INR, international normalized ratio; IQR, interquartile range; $h$, hour; min, minute.

12 hours; range, 10 minutes to 96 hours). Of the 139 affected patients who presented within 24 hours after bitten, monitoring for 24,48 , and 49 hours enabled detection of systemic effects in $94.2 \%, 99.3 \%$, and $100.0 \%$, respectively (Figure 3).

Allergic reactions to venom occurred in four patients (2.4\%). Symptoms of allergic reaction included anaphylaxis (three patients) and hypotension (one patient). Other complications included acute coronary syndrome (one patient), rhabdomyolysis (two patients), hemolysis (one patient), and uterine contractions (one patient). The case who developed acute coronary syndrome was a 53-year lady with no known underlying medical condition. She reported new onset chest pain 6 hours after bitten by MPV at both legs. Her electrocardiogram showed ST depression in lead V3-V6. Her troponin- $\mathrm{T}$ was $1000 \mathrm{ng} / \mathrm{L}$ (normal range less than $100 \mathrm{ng} / \mathrm{L}$ ). Her chest $\mathrm{x}$-ray showed mild cardiomegaly. She was diagnosed to have non-ST elevation myocardial infarction, with newly diagnosed hypertension and dyslipidemia by the cardiologist. She was suspected to have some degree of coronary syndrome before this episode. She received aspirin, clopidogrel, atorvastatin and subcutaneous enoxaparin for 5 days. She was later discharged with aspirin, atorvastatin, and clopidogrel. Her highest INR was 1.1. Her lowest platelet count was $114,000 / \mu \mathrm{L}$, which gradually increased without antivenin.

\section{Management}

One-hundred forty-four patients $(86.2 \%)$ received antivenin. In total, 315 courses (1195 vials) of antivenin were administered, including 296 courses of monovalent MPV antivenin and 19 courses of polyvalent hematotoxic snake antivenin. The median quantity of antivenin treatment per patient receiving antivenin was two courses (IQR, 1-3; range, 1-7) or six vials (IQR, 4-11; range, 1-28). The most common indication for antivenin was prolonged VCT. Two hundred and sixty-nine courses of antivenin (85.4\% of the 315 courses) were considered appropriate administration (Table 5); 22 courses of antivenin $(7.0 \%)$ were administered without Thai Red Cross indication. Other treatments included antibiotics (130 patients), surgical intervention (32 patients included 20 cases with wound debridement, 10 cases with fasciotomy, and 2 cases with both debridement and fasciotomy), and blood components (19 patients).

Adverse reactions related to antivenin administration occurred in 50 patients $(34.7 \%$ of the 144 patients who received antivenin) -65 courses of antivenin $(20.6 \%$ of the 315 antivenin courses in this study). Common adverse reactions were rash, chest tightness, lung wheezing, and vomiting (Table 6). Anaphylaxis occurred in 29 patients 
Cases (n)

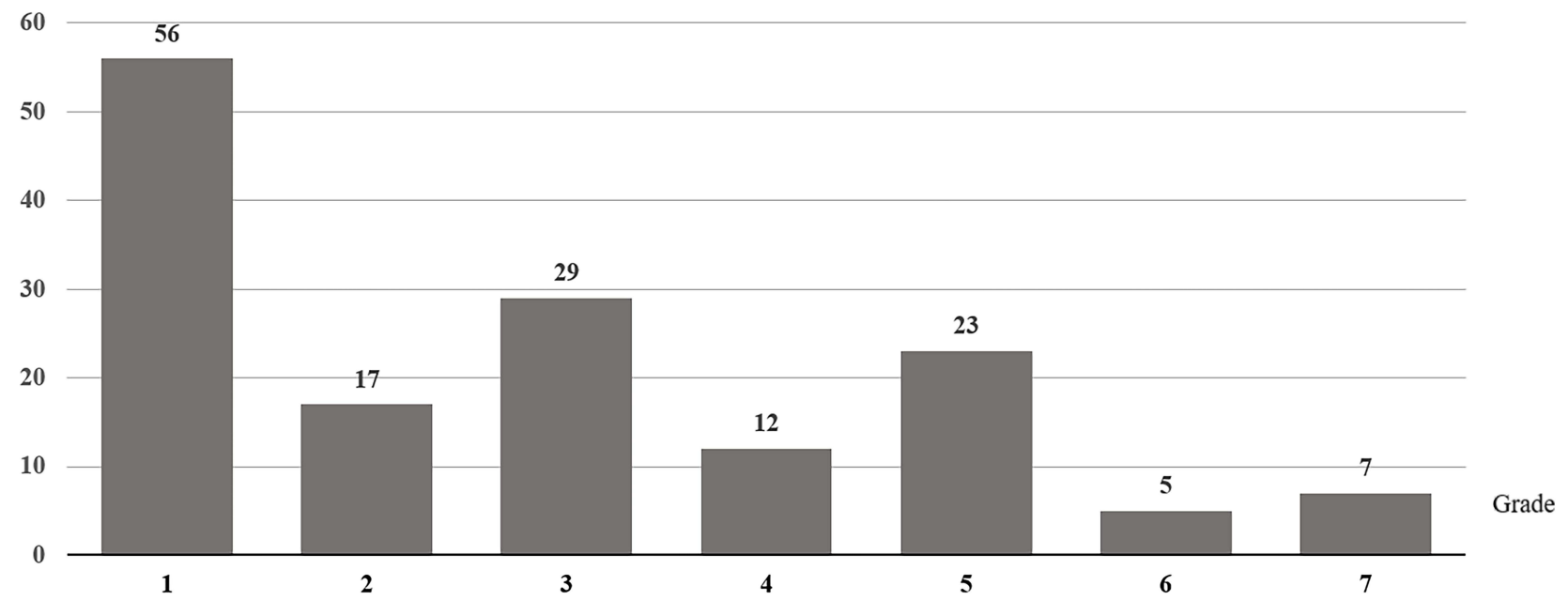

Figure 2 Grading of local effects after Malayan pit viper bite reported to Ramathibodi Poison Center during July 2016 to June 2018.

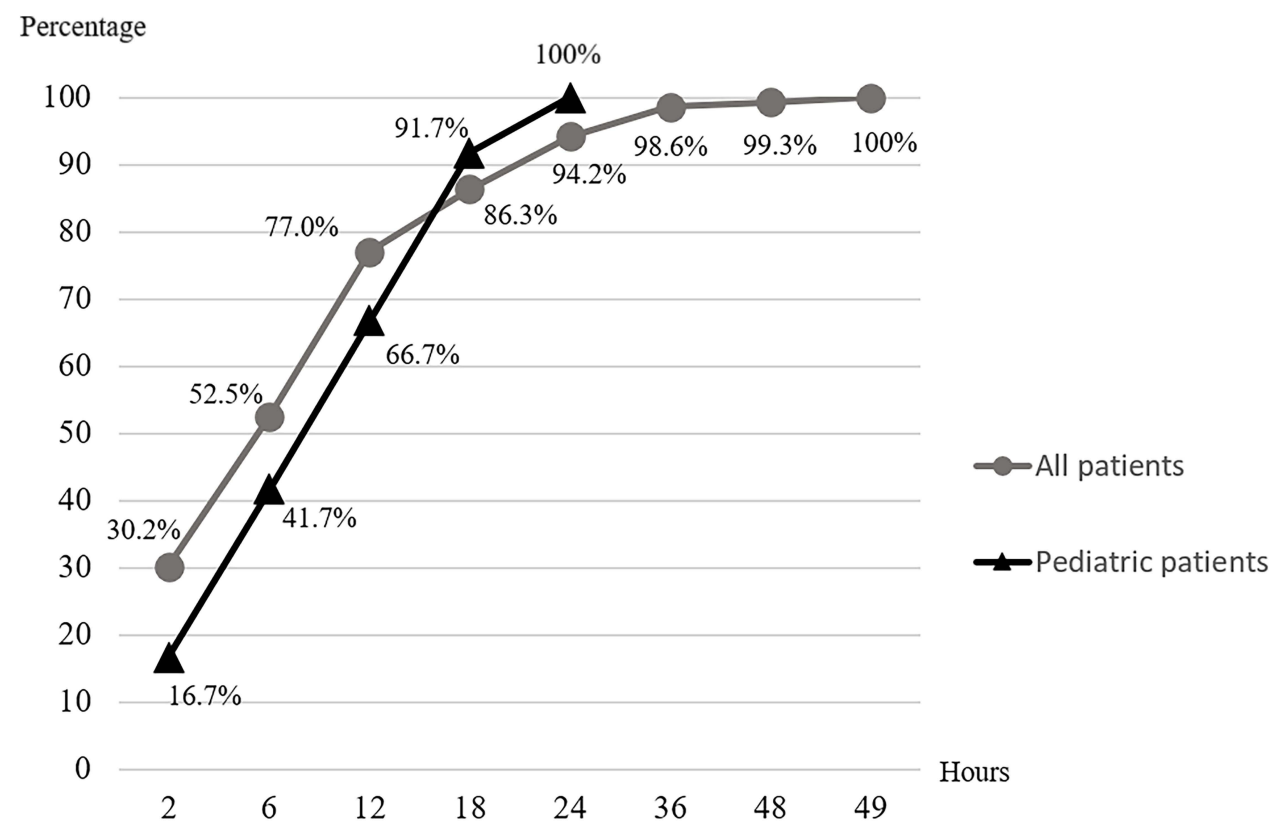

Figure 3 Cumulative percentage of cases with systemic effect over time.

( $20.1 \%$ of the 144 patients who received antivenin) -30 courses of MPV antivenin and one course of polyvalent hematotoxic snake antivenin.

Recurrent bleeding disorders after antivenin occurred in 21 patients $(14.6 \%$ of the 144 patients who received antivenin); these included prolonged VCT (16 patients), unclotted 20-minute whole blood clotting time (four patients), INR $>1.2$ (four patients), and platelet count $<50,000 / \mu \mathrm{L}$ (two patients). Median time of recurrence was 41 hours after the initial bite (IQR, 35-60 hours; range, 17-92 hours) and 18 hours after treatment with antivenin (IQR, 13-30 hours; range, 8-46 hours). No patients with recurrence exhibited systemic bleeding.

\section{Outcomes}

Median length of stay was 3 days with no re-admission (IQR, 2-5; range, 1-29). All patients who received antivenin recovered from bleeding disorder. One patient died of rhabdomyolysis complications after full recovery from systemic effects. One patient underwent finger amputation. 
Table 5 Antivenin Administration for Patients with Malayan Pit Viper Envenomation Reported to Ramathibodi Poison Center ( $\mathrm{n}$ $=315$ Courses)

\begin{tabular}{|l|c|}
\hline Antivenin Administration & Course (\% of $\mathbf{3}$ I 5 Courses) \\
\hline Indications & $212(67.3)$ \\
Prolonged VCT & $73(23.2)$ \\
Unclotted 20WBCT & $30(9.5)$ \\
Platelet $<50,000 \mathrm{mcL}$ & $42(13.3)$ \\
INR $>$ I.2 & $14(4.4)$ \\
Systemic bleeding & $1(0.3)$ \\
Operative management & $\mathbf{2 6 9}(85.4)$ \\
\hline Appropriate administration & $293(93.0)$ \\
Correct indication & $305(96.8)$ \\
Appropriate dosage & $300(95.2)$ \\
Appropriate duration of infusion &
\end{tabular}

Abbreviations: VCT, venous clotting time; 20WBCT, 20-minute whole blood clotting test; INR, international normalized ratio.

Table 6 Allergic Reaction to Antivenin in Patients with Malayan Pit Viper Envenomation Reported to Ramathibodi Poison Center ( $n=315$ Courses)

\begin{tabular}{|l|c|}
\hline Symptoms & Course (\% of 3 I5 Courses) \\
\hline Skin manifestation & $47(14.9)$ \\
Rash & $6(1.9)$ \\
Pruritus & $2(6.3)$ \\
Angioedema & \\
\hline Gastrointestinal manifestation & $9(2.9)$ \\
Nausea and vomiting & $1(0.3)$ \\
Abdominal pain & \\
\hline Respiratory manifestation & $12(3.8)$ \\
Dyspnea & $14(4.4)$ \\
Wheezing lung & $19(6.0)$ \\
Chest tightness & $5(1.6)$ \\
Hypoxia & \\
\hline Cardiovascular manifestation & $13(4.1)$ \\
\hline Hypotension & \\
\hline
\end{tabular}

Of the 138 patients who initially received MPV antivenin, four did not match any indication. One-hundred thirty-four patients initially received MPV antivenin for treatment of bleeding disorder. Fifty-five patients $(41.0 \%$ of 134 patients) recovered from bleeding disorder after one course of MPV antivenin. Thirty-eight patients $(28.4 \%$ of 134 patients) recovered from bleeding disorder after two courses of MPV antivenin. One patient recovered after receiving one course of MPV antivenin followed by one course of polyvalent hematotoxic snake antivenin. Thirty- nine patients $(29.1 \%$ of 134 patients) received more than 2 courses of antivenin.

Six patients initially received polyvalent hematotoxic snake antivenin. Six of them either brought the snake carcasses ( 3 patients) or were able to recognize the snakes (3 patients). Four patients recovered after one course. The rest recovered after receiving two and three courses of polyvalent hematotoxic snake antivenin.

\section{Special Populations}

Seventeen children with MPV envenomation were reported to the RPC during the study period; their median age was 9 years (IQR, 9-11 years; range, 1-14 years). Sixteen patients (94.1\% of the 17 children) experienced local effects. Thirteen patients (76.5\%) experienced systemic effects including prolonged VCT (11 patients), unclotted 20-minute whole blood clotting time (three patients), platelet count $<50,000 / \mu \mathrm{L}$ (one patient), INR $>1.2$ (six patients), and systemic bleeding (one patient). No patients exhibited dry bite. The median durations until onset of each abnormal test result and systemic bleeding are shown in Table 7. The median onset of bleeding disorder was 11 hours (IQR, 5-15 hours; range, 0.5-59 hours). Of the 12 affected patients who presented within 24 hours after bitten, monitoring for 12, 18, and 24 hours enabled detection of systemic effects in $66.7 \%, 86.3 \%$, and $100.0 \%$, respectively (Figure 3 ). Twenty courses of antivenin were prescribed for 13 patients (76.5\%). Adverse reactions from antivenin occurred in six patients $(46.2 \%$ of the 13 children who received antivenin), including nine courses of antivenin (45.0\% of the 20 antivenin courses administered to children in this study). Operations were performed in six of the 17 children (35\%) included 5 wound debridement, and one fasciotomy. The median

Table 7 Onset of Systemic Toxicity in 17 Pediatric Cases with Malayan Pit Viper Envenomation Reported to Ramathibodi Poison Center

\begin{tabular}{|l|c|}
\hline Systemic Effects & $\begin{array}{c}\text { Median Onset of } \\
\text { Abnormality (IQR; Range) }\end{array}$ \\
\hline $\begin{array}{l}\text { Prolonged VCT } \\
\text { Unclotted 20WBCT }\end{array}$ & $\begin{array}{c}2.5 \mathrm{~h}(5-18.5 \mathrm{~h}, 30 \mathrm{~min}-32.5 \mathrm{~h}) \\
\text { INR }>\text { I.2 }\end{array}$ \\
$\begin{array}{l}\text { Overall onset of systemic effects } \\
\text { I } 7 \mathrm{~h}(\mathrm{I}-15.5-20.75 \mathrm{~h}, 30 \mathrm{~min}-4 \mathrm{I} \mathrm{h}) \\
\mathrm{II} \mathrm{h}(5-15 \mathrm{~h} ; 0.5-59 \mathrm{~h} *)\end{array}$ \\
\hline
\end{tabular}

Note: *One patient presented with gingival bleeding and low platelet count at 59 $\mathrm{h}$ after bitten.

Abbreviations: VCT, venous clotting time; 20WBCT, 20 minutes whole blood clotting test; INR, international normalized ratio; IQR, interquartile range; h, hour; min, minute. 
length of stay was 3 days (IQR, 2-5 days; range, 1-13 days).

Three patients had a history of warfarin use without antiplatelet therapy, due to underlying heart disease; all had prolonged INR $>1.5$ during the first test. Antivenin was prescribed for two patients, with a median of two courses of antivenin. The third patient was observed without antivenin treatment. At the time of discharge, the INR values for all three patients were within the target range for warfarin treatment.

One patient had been taking aspirin because of heart condition; the patient exhibited prolonged VCT with a normal platelet count and normal INR. This patient was treated with antivenin and exhibited full recovery at discharge. Another patient had been taking both aspirin and warfarin; the patient experienced hematemesis and received three courses of antivenin and blood components. This patient exhibited INR of 1.2 at the time of discharge.

\section{Discussion}

This retrospective study revealed that MPV bites occur in all regions of Thailand. However, a previous study by Wongtongkam et al revealed no MPV bites in the central and north eastern regions. ${ }^{4}$ These discrepant findings might be explained by changes in MPV distribution due to modifications of both climate and landscape. ${ }^{13} \mathrm{MPV}$ envenomation is most commonly considered to be endemic in the southern region. ${ }^{3,6}$ In studies by Reid et al and Wongtongkam et al, rubber tappers (a primary occupation in the southern region) were found to be most commonly bitten by MPVs. ${ }^{2,3}$ The peak snake bite season is reported in May, which is early monsoon season. ${ }^{3}$ Our study showed that $3 \%$ of patients exhibited dry bite, which was similar to the rate in Laos, described by Vongphoumy et al $(2.5 \%){ }^{4}$

In the present study, $88.0 \%$ of patients with MPV envenomation exhibited bleeding disorder, which was higher than the proportions reported by Viravan et al (33.8\%), Wongtongkam et al (46.4\%), and Kraisawat et al (38.6\%). ${ }^{1,3,14}$ Notably, more laboratory parameters were recorded in our study - particularly VCT - compared with previous studies. In the current practice, abnormal VCT results comprise $>20$ minutes; this value is lower than the threshold used by Wongtongkam et al (VCT $>30$ minutes). Differences in reported parameters and bleeding disorder criteria among studies may explain the differences in proportions of patients with systemic toxicity. Additionally, VCT is subjected to methodological and analytical errors yielding false-positive results for hypofibrinogenemia caused by pit vipers. ${ }^{15}$ Other methods with better sensitivity and specificity should be utilized to detect bleeding disorder in MPV envenomation such as 20minute WBCT and INR. ${ }^{16}$ More appropriate diagnostic tools would also reduce inappropriate antivenin administration.

In our study, the median time to bleeding disorder onset was 6 hours after MPV bite. The longest duration until onset of bleeding disorder in our study was 49 hours. Ho et al previously reported that the onset of bleeding disorder may occur up to 72 hours after MPV bite. ${ }^{17}$ The only clotting tests used in that study were VCT and fibrinogen concentration. The use of multiple types of bleeding tests may have resulted in earlier detection of systemic toxicity in our study.

In our study, antivenin was administered in $88 \%$ of patients, compared with $58 \%$ in the Laos study. This difference in treatment may be related to the different indications for antivenin administration. Indications in the Laos study did not include abnormal VCT or whole blood clotting time; additionally, INR was required to be $>5 .{ }^{4}$ The median volume of antivenin in the present study was six vials per case; this was similar to the finding in a previous study in Thailand (approximately 4-9 vials). ${ }^{3}$ Proteomic analysis showed that MPV venom components predominantly metalloproteinases, serine proteases, L-amino acid oxidase, and phospholipase A2, etc. causing hemorrhage, coagulopathy, thrombocytopenia, and tissue necrosis - were effectively neutralized by MPV antivenin. ${ }^{8}$ This corresponded to our finding that bleeding disorders recovered fully after antivenin. However, the efficacy of antivenin against local effects is still questionable; some patients still required surgical intervention after antivenin therapy in our results and previous studies. ${ }^{3,14}$

Our study revealed $10 \%$ severe allergic reaction to antivenin or anaphylactic reaction, which is lower than the rate reported by Vongphoumy et al. (30\%). ${ }^{4}$ The reason for different rates of adverse reactions remains unclear but could be due to a hospital-based selection bias, as Thai Universal Health Coverage makes healthcare more accessible and economical. Recurrent systemic toxicity was uncommon (14.6\%). Recurrent coagulopathy may occur due to a reduction in circulating antivenin level or reversible binding of antivenin but may not be directly related to the elimination half-life of antivenin. ${ }^{18}$

For limb edema, our poison center would initially suggest supportive treatment such as adequate limb 
elevation and local wound care to avoid premature diagnosis of compartment syndrome and risk the patients for surgery which is rarely indicated.

The mortality rate of patients with MPV bite in our study was $<1 \%$, similar to the finding in a previous study by Wongtongkam et al; in that study, deaths occurred as a result of systemic bleeding (eg, intracranial hemorrhage or hemorrhagic shock from hematemesis). ${ }^{3}$ In our study, one patient died of rhabdomyolysis after recovery from systemic bleeding.

Among pediatric patients (aged $<15$ years), none exhibited dry bite. The proportion of pediatric patients with systemic toxicity did not differ from the overall population. However, all systemic effects were detected earlier in children than in adults ( 24 hours vs 49 hours). Notably, pediatric patients exhibited a higher rate of allergic reaction after antivenin treatment; this might be caused by a greater amount of antivenin relative to body weight. In addition, there was a high rate of surgical intervention (35\%; six of 17 patients), compared with a previous study in pediatric patients $(3.5 \%){ }^{10}$

A previous case report described abruptio placentae and fetal death following MPV bite. ${ }^{19}$ In our study, vaginal bleeding only occurred in one pregnant woman, who completely recovered after antivenin and supportive treatment.

As a retrospective study of poison center data, which was voluntarily reported, there were some limitations including the potential for missing data and selection bias. Patients reported to a poison control center may exhibit more severe envenomation. Information regarding clinical manifestations (both history and physical examination) and diagnosis (such as compartment syndrome) reported by primary physicians. The Ramathibodi Poison Center provides guidelines for treatment, but the decisions for treatments are made by attending physicians. There was no routine documentation of some aspects such as pain score, wound culture, and hemoculture results. This study was based on Thai MPV envenomation; thus, the results may be not generalizable to other regions. Patients were reported from all regions of Thailand; the findings in this study might have been affected by variations in practice, laboratory methods, and reference values among hospitals.

\section{Conclusions}

MPV envenomation results in local and systemic effects. Dry effects from MPVs are rare. Most systemic effects comprised abnormal clotting test results, and most patients experienced onset of bleeding disorder within 48 hours.

\section{Disclosure}

The authors report no conflicts of interest.

\section{References}

1. Viravan C, Looareesuwan S, Kosakarn W, et al. A national hospital-based survey of snakes responsible for bites in Thailand. Trans R Soc Trop Med Hyg. 1992;86(1):100-106. doi:10.1016/ 0035-9203(92)90463-M

2. Reid HA, Thean PC, Chan K, et al. Clinical effects of bites by Malayan viper (Ancistrodon rhodostoma). Lancet. 1963;281 (7282):617-621. doi:10.1016/S0140-6736(63)91268-6

3. Wongtongkam N, Wilde H, Sitthi-Amorn C, et al. A study of 225 Malayan pit viper bites in Thailand. Mil Med. 2005;170:342-348. doi:10.7205/MILMED.170.4.342

4. Vongphoumy I, Chanthilat P, Vilayvong P, et al. Prospective, consecutive case series of 158 snakebite patients treated at Savannakhet provincial hospital, Lao People's Democratic Republic with high incidence of anaphylactic shock to horse derived $F(a b ') 2$ antivenom. Toxicon. 2016;117:13-21. doi:10.1016/j.toxicon.2016.03.011

5. Looareesuwan S, Viravan C, Warrell DA. Factors contributing to fatal snake bite in the rural tropics: analysis of 46 cases in Thailand. Trans $R$ Soc Trop Med Hyg. 1988;82(6):930-934. doi:10.1016/0035-9203(88)90046-6

6. Tan NH, Ponnudurai G. The toxinology of Calloselasma rhodostoma (Malayan pit viper) venom. J Toxicol Toxin Rev. 1996;15(1):1-17. doi:10.3109/15569549609080104

7. Rojnuckarin P. Snakebite-induced coagulopathy and bleeding disorders. In: Kini RM, Clemetson KJ, Markland FS, McLane MA, Morita T, editors. Toxins and Hemostasis: From Bench to Bedside. Dordrecht: Springer Netherlands; 2010:699-710.

8. Tang ELH, Tan NH, Fung SY, Tan $\mathrm{CH}$. Comparative proteomes, immunoreactivities and neutralization of procoagulant activities of Calloselasma rhodostoma (Malayan pit viper) venoms from four regions in Southeast Asia. Toxicon. 2019;169:91-102. doi:10.1016/j. toxicon.2019.08.004

9. Rojnuckarin P, Suteparak S, Sibunruang S. Diagnosis and management of venomous snakebites in Southeast Asia. Asian Biomed. 2017;6(6):795-805.

10. Laohawiriyakamol S, Sangkhathat S, Chiengkriwate $P$, Patrapinyokul S. Surgery in management of snake envenomation in children. World J Pediatr. 2011;7(4):361-364. doi:10.1007/s12519011-0282-8

11. Rojnuckarin P, Mahasandana S, Intragumtornchai T, Swasdikul D, Sutcharitchan P. Moderate to severe cases of green pit viper bites in Chulalongkorn hospital. Thai J Hematol Transfus Med. 1996;6 (3):199-205.

12. Sampson HA, Muñoz-Furlong A, Campbell RL, et al. Second symposium on the definition and management of anaphylaxis: summary report second national institute of allergy and infectious disease/food allergy and anaphylaxis network symposium. J Allergy Clin Immunol. 2006;117(2):391-397. doi:10.1016/j.jaci.2005.12.1303

13. Zacarias D, Loyola R. Climate change impacts on the distribution of venomous snakes and snakebite risk in Mozambique. Clim Change. 2019;152(1):195-207. doi:10.1007/s10584-018-2338-4

14. Kraisawat K, Promwang N. Duration after Malayan pit viper bite to detect coagulopathy in Songklanagarind hospital. J Health Sci Med Res. 2020;38(2):93-101.

15. Wedasingha $\mathrm{S}$, Isbister $\mathrm{G}$, Silva A. Bedside coagulation tests in diagnosing venom-induced consumption coagulopathy in snakebite. Toxins. 2020;12(9):583. doi:10.3390/toxins12090583 
16. Thongtonyong N, Chinthammitr Y. Sensitivity and specificity of 20-minute whole blood clotting test, prothrombin time, activated partial thromboplastin time tests in diagnosis of defibrination following Malayan pit viper envenoming. Toxicon. 2020;185:188-192. doi:10.1016/j.toxicon.2020.07.020

17. Ho M, Warrell DA, Looareesuwan S, et al. Clinical significance of venom antigen levels in patients envenomed by the Malayan pit viper (Calloselasma rhodostoma). Am J Trop Med Hyg. 1986;35 (3):579-587. doi:10.4269/ajtmh.1986.35.579
18. Ho M, Silamut K, White NJ, et al. Pharmacokinetics of three commercial antivenoms in patients envenomed by the Malayan pit viper, Calloselasma rhodostoma, in Thailand. Am J Trop Med Hyg. 1990;42 (3):260-266. doi:10.4269/ajtmh.1990.42.260

19. Hanprasertpong J, Hanprasertpong T. Abruptio placentae and fetal death following a Malayan pit viper bite. J Obstet Gynaecol Res. 2008;34(2):258-261. doi:10.1111/j.1447-0756.2008.00766.x

\section{Publish your work in this journal}

Therapeutics and Clinical Risk Management is an international, peerreviewed journal of clinical therapeutics and risk management, focusing on concise rapid reporting of clinical studies in all therapeutic areas, outcomes, safety, and programs for the effective, safe, and sustained use of medicines. This journal is indexed on PubMed Central, CAS,
EMBase, Scopus and the Elsevier Bibliographic databases. The manuscript management system is completely online and includes a very quick and fair peer-review system, which is all easy to use. Visit http://www.dovepress.com/testimonials.php to read real quotes from published authors. 